ALÉTHEIA

$2015,3(1) 21-27$

Recibido: 16-06-2015

Aceptado: 08-07-2015

Palabras clave: gestión, gestión de le educación, administración, dirección y habilidades $y$ competencias directivas.

Keywords: management, cducational management, administration, management skills and management competencies.

César Bravo Macedo UTP macedobravocesar@gmail.com

\section{Reflexiones sobre la gestión educativa y las competencias directivas para el desarrollo de la educación}

\section{Analysis of management in education and guideline competences for education development}

\section{Cesar Bravo Macedo}

RESUMEN. El presente trabajo de investigación contiene un análisis descriptivo sobre el significado de la gestión y la ditección en el ámbito educativo, en el contexto de las normas y disposiciones existentes para directores de centros educativos en el Perú. Este análisis se realiza en contraste con las teotias de la administración y con los conceptos modernos sobre esta especialidad.

Como tesultado de este análisis, se evidencian temáticas en los que los directivos tendían que desarrollar competencias para ejercer el cargo directivo. Asimismo, se propone otras competencias transversales para estos, tomando en considetación los cambios disruptivos que se producen en la globalización para la administración de las organizaciones. No siendo ajenas las organizaciones educativas a estos cambios, la reflexión permite, además, ver con claridad las responsabilidades directivas que tendrian que asumir los directivos en el futuro para "gestionar" los centros educativos. Pata el presente trabajo de investigación se ha recurrido a filentes normativas vigentes existentes en el Ministerio de Educación (MINEDU), entre ellos, documentos informativos y normativos que se emiten en dicho Ministerio, y que sirven para la capacitación de directivos; asimismo, bibliografía especializada de administración de las organizaciones, lo que ha permitido tener una apreciación más objetiva pata la reflexión en este tema.

ABSTRACT. The present reseatch includes a descriptive analysis of the meaning of management in education, in the context of the existing regulations and guidelines for school headteachers in Peru. The analysis cartied out is contrasted with management theories and modern concepts in this field.

As a result of this analysis, it is evidenced that school authorities should develop competencies to assume managetial positions. In addition, several other ttanswersal competencies ate suggested, considering the disruptive changes caused by globalization for maragement, and the fact that educational organizations are unable to avoid such changes. In addition, reffection enables a clear view of the managerial responsibilities that school authorities should undertake in the future to "manage" schools.

This research has used existing normative sources from the Ministry of Education (MINEDU), such as informative and normative documents issued at this Ministry and used for school authority training, as well as specialized bibliographical sources on organizational management, leading to a more objective appreciation for reflecting on this issue. 


\section{UNIFÉ-EPG}

\section{Introducción}

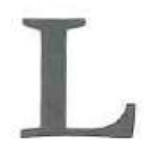

os principales actores en las instituciones educativas son los docentes y los directores de los centros educativos. En cada caso, se han planteado diversas propuestas para mejorar sus competencias, con el fin de asegurar la calidad educativa y principalmente transformar la educación en el Perú. Alrededor de la literatura para la gestión educativa y para la labor de los directores de los centros educativos, se mencionan un conjunto de términos relacionados con la gestión y administración de la educación con la finalidad de que estos actores interioricen las responsabilidades de su gestión conforme las normas emitidas por el MINEDU para directores de centros educativos.

Vista la gestión desde la perspectiva de la administración, se puede identificar un conjunto de términos relacionados con temas vinculados a la gestión y a la administración de la educación, que no permiten tener un panorama claro de las competencias directivas que les ayuden a comprender adecuadamente la gestión en educación, en la mayoría de casos, por la naturaleza de la especialidad (educación) que tienen los directores de Instituciones Educativas (II.EE).

La administración de las organizaciones educativas, entre otros tipos de organizaciones, son aún más complejas por el tipo de servicio y/o "producto" que "elabora"; por ello, es necesario analizar la gestión educativa desde su complejidad.

Tomando en consideración el escenario antes referido, la presente investigación descriptiva contiene el análisis de los conceptos de gestión y de dirección existentes en las normas vinculadas al MINEDU versus los principios de la ciencia de la administración Para ello, se han abordado temas como la gestión entendida desde el MINEDU, la gestión y la dirección comprendida desde la Ciencia de la Administración, la Complejidad de las Organizaciones Educativas y finalmente las Competencias y Habilidades directivas en la educación.

\section{Significado de Gestión}

Para el análisis se ha tomado información sobre el origen de la palabra "gestión", el concepto relacionado con la administración, y por otro lado, los documentos del MINEDU que expresan diversas interpretaciones respecto de la gestión en documentos relevantes que se otorgan a docentes y directores de II.EE.

Según la real Académica Española (RAE 2014):

1. f. Acción y efecto de gestionar.

2. f. Acción y efecto de administrar.

Según otras fuentes:

- (...) llevar a cabo diligencias que hacen posible la realización de una operación comercial o de un anhelo cualquiera (definición/gestión).

- (...) un conjunto de acciones u operaciones relacionadas con la administración y dirección de una organización (significados.com/gestión).

\section{Según documentos del MINEDU}

\begin{tabular}{|c|c|c|}
\hline FUENTE & SIGNIFICADO & DOCUMENTO \\
\hline A & $\begin{array}{l}\text { (...) función dirigida a generar y } \\
\text { sostener en el centro educativo tanto las } \\
\text { estructuras administrativas y pedagógicas, } \\
\text { como los procesos internos de naturaleza } \\
\text { democrática, equitativa y eficiente,... }\end{array}$ & RM 168-2002-ED \\
\hline B & $\begin{array}{l}\text { (..) conjunto de estrategias diferenciadas } \\
\text { dirigidas a la solución de problemas, que } \\
\text { deben ser claramente identificados y } \\
\text { caracterizados. } \\
\text { (...) el modelo de gestión que } \\
\text { proponemos aspira a objetivos de } \\
\text { corto y mediano plazos; propone un } \\
\text { mayor número de alternativas posibles } \\
\text { para un futuro más remoto debido a la } \\
\text { incertidumbre (...) }\end{array}$ & $\begin{array}{l}\text { Unidad de } \\
\text { Capacitació n } \\
\text { Docente (UCAD 2, } \\
2000 \text { ) }\end{array}$ \\
\hline C & $\begin{array}{l}\text { (...) capacidad de dirigir la organización } \\
\text { de los recursos en los centros educativos } \\
\text { y la aplicabilidad de herramientas, } \\
\text { técnicas y métodos para el logto eficaz } \\
\text { de resultados (...) }\end{array}$ & $\begin{array}{l}\text { Manual de Redes } \\
\text { Educativas Rurales } \\
\text { (UDECE, 2001) }\end{array}$ \\
\hline $\mathrm{D}$ & $\begin{array}{l}\text { (...) uso de cinco nuevas estrategias que } \\
\text { convergen para innovar la organización } \\
\text { y funcionamiento del centro educativo: } \\
\text { el dominio personal, modelos mentales, } \\
\text { pensamiento sistémico, visión } \\
\text { compartida y aprendizaje en equipo. } \\
\text { (UCAD 1, 2000) [Clarificar y formular] } \\
\text { propuestas de trabajo en conjunto (...). } \\
\text { (UCAD 2,2000) }\end{array}$ & $\begin{array}{l}\text { Unidad de } \\
\text { Capacitación } \\
\text { Docente (UCAD) }\end{array}$ \\
\hline
\end{tabular}


Alétheia 2015, 3 (1)

\begin{tabular}{|c|c|}
\hline PUINTE: & SIGNIICADO \\
\hline $\mathrm{E}$ & 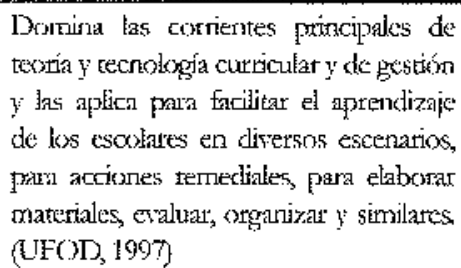 \\
\hline$l^{i}$ & 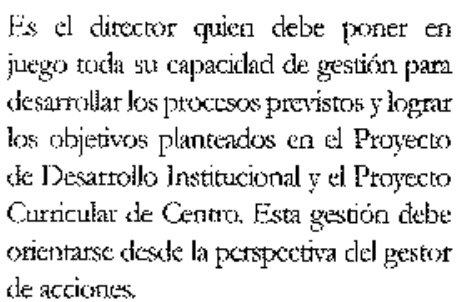 \\
\hline
\end{tabular}

G

Por esta kazin, el director o directorn alcanza importancia relevante no solamente con relación a sus docentes, sino tarobién con los estudiantes, padres $y$ madres de familia, debiendo ser capnz de apryar decididumente el proceso de cambio en la oncepción y práctica education que leneficintán a los esiudiantes (UCAD 1, 2000)

Los centros educarivos dienen autonomía pata diseinar inclicadorex, criterios e insurmentos de autoevaluación pam medir los aprendizajes $y$ la gestión educativa, con la frnaliclad de que se tomen decisiones oportunas $y$ pertinentes en la mejora de la calidad y hapuidad.

$J$

Sisterna de Gention Educativa:

Ln clesafó para el sisterna de gestión educativa en que kos procesos de phneaniento, presupuesto, monitoreo $y$ evaluacion estén articulados entre si para gatantivat que in asignación de recutsos rengra oomo referencia el logro de los resultactos.

\section{Curriculo, Tecnologia y Gestión:}

\section{DOCUMENTO}

El Currículo Básico de Formación Ducente plantea (MDNEDU, 2003)

Unichad

Desartollo

Curricular y

Recursos Educativos

(DDCREFS, 2002) proceson de grestión institucional |... lider scriala en sus guias de lox ejuipos docentes (UCG, 1999) de capacitación que al modek de gestion institucional, admirnistrativia $y$ pexlagriogica

Unikiaxl dc

Capacitación Docente (UCAD)

VI cido: Gestión Exhucaria-Gestión del ath (diseño reglamentos, ambientación, organizacion) - Gertión inctitucional (I.jderazso y gerencia projectos de grestión, proyectos de desarrollo instrincional) - Gersión del Sistema (legishacion, otganización ý supcetwisión) pain la gesión cescentralizada de ha eclucación "Componentes de soporte Institucional" (AINLDU, 2013)

Direccion Nacional de: Formación y Capacitacion Doctnte (NINEDU, 2003)

\begin{tabular}{|c|c|c|}
\hline IVUENTT? & SICNIJHICADO & DOCUMENTO \\
\hline $\mathrm{L}$ & 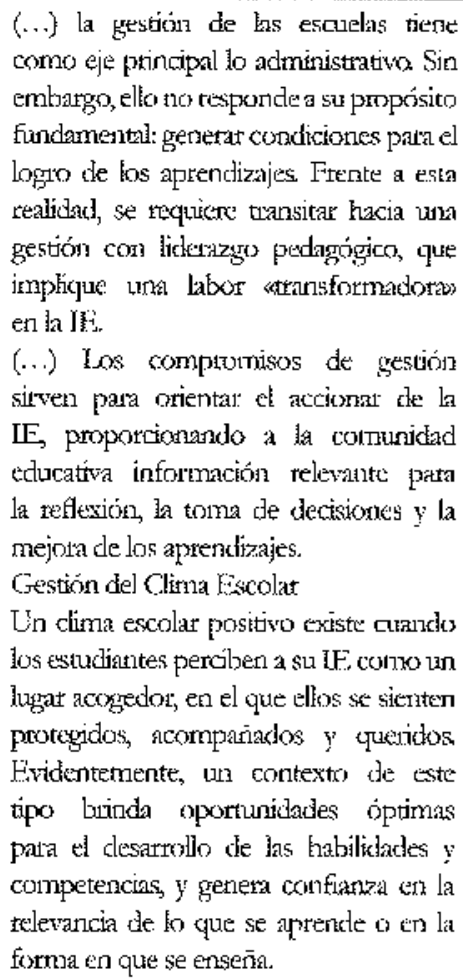 & $\begin{array}{l}\text { Fasciculo "Gestión } \\
\text { escolar Centrada en } \\
\text { los Aptendizajes" } \\
\text { (MINEDU, 2003) }\end{array}$ \\
\hline
\end{tabular}

\section{Según la teoría de la administración}

Si tomamos en consideración que la gestión es vinculante con la teoría de la adnuinistración y esta se sustenta en el Proceso Administrativo (Planeación, Organización, Dirección y Control) además de las nuevas concepciones de la administración, los directivos de las organizaciones educativas, independientemente de sus objetivos administrativos y/o pedagógicos, tendrán que desarrollar competencias para ejecutar este proceso administrativo en sus características de secuencialidad, cíclica, dinámica e interactiva.

$Y$ si tomamos en cuenta las nuevas tendencias

A continuación, se presentan definiciones de acreditados autores que coinciden en los fundamentos de la administración referidos anteriormente:

Chiavenato, I. (2004) señala que "es el proceso de planear, otganizat, dirigit y controlar el uso de los recursos para lograt los objetivos otganizacionales". 


\section{UNIFÉ - EPG}

Koontz, H. (2004) define que "es el proceso de diseñar y mantener un entorno en el que, trabajando en grupos, los individuos cumplan eficientemente objetivos específicos"

Robbins, S. y De Cenzo, D. (2003) señala lo siguiente "es el proceso de realizar actividades y terminarlas eficientemente con y a través de otras personas." $[\ldots]$

"Estas funciones son tradicionalmente clasificadas como: planeación, organización, dirección y control"

\section{Resultados del Análisis}

\section{Sobre el significado de la gestión versus la administración}

- La gestión constituye parte esencial de la administración como ciencia, por tanto, la construcción de información, alrededor de esta palabra, debe estar conformada respetando los principios de la administración, así como de la teoria vigente de esta ciencia.

Sobre la interpretación de la gestión por el MINEDU

- De la información recopilada en algunos de los documentos del MINEDU, se evidencia una variedad de significancias, alrededor de la palabra gestión, las mismas que tienen un componente negativo y otro positivo, que se explica a continuación:

a. Los docentesydirectivos del sistemaeducativo en el Perú, podrían tener confusiones respecto de su significado, debido a que los documentos referenciados anteriormente evidencian una serie de interpretaciones que, si bien es cierto están relacionadas, generan confusión para el manejo de los términos al momento de interpretar la norma como parte de sus responsabilidades. Asimismo, se espera que exista coherencia entre las disposiciones (normas) y las competencias de los directivos.

b. El aspecto positivo de este análisis es que se identifica un conjunto de competencias a las que se pueden orientar las capacitaciones de los directivos con el fin de que adquieran habilidades para cumplir con las exigencias del MINEDU para la gestión educativa. Al respecto, la condición actual de estas competencias es relativamente adecuada, considerando que gran parte de los cargos están ocupados por especialistas en educación.

c. Tomando en consideración los temas de administración observados alrededor de la "gestión" en los documentos del MINEDU (Ver Tabla 1, para identificar la fuente), se ha elaborado un listado de estas temáticas vinculadas con la gestión, con el fin de identificar las competencias que deberíamos lograr para el directivo de una II.EE. estas se mencionan a continuación:

Tabla 2

Temas para la competencias en gestión

\begin{tabular}{clc}
\hline $\mathrm{N}^{\circ}$ & Tema para la competencia en gestión educativa o escolar & Fuente $^{*}$ \\
\hline 1 & Administración por objetivos & $\mathrm{B}$ \\
2 & Autocontrol y Autoconocimiento & $\mathrm{D}$ \\
3 & Comunicación Efectiva y Asertiva & $\mathrm{H}$ \\
4 & Control y Evaluación & $\mathrm{I}$ \\
5 & Dirección y Organización & $\mathrm{C}$ \\
6 & Diseño de Indicadores & $\mathrm{I}$ \\
7 & Elaboración Proyectos & $\mathrm{Ky} \mathrm{L}$ \\
8 & Gerencia & $\mathrm{K}$ \\
9 & Gestión de Procesos & $\mathrm{Fy} \mathrm{G}$ \\
10 & Gestión Estratégica & $\mathrm{B}$ \\
11 & Liderazgo & $\mathrm{K}$ \\
12 & Manejo de Escenarios & $\mathrm{E}$ \\
13 & Organización & $\mathrm{A}$ \\
14 & Pensamiento Sistémico & $\mathrm{D}$ \\
15 & Planeamiento y Presupnesto & $\mathrm{J}$ \\
16 & Toma de Decisiones & $\mathrm{L}$ \\
17 & Clima Laboral & $\mathrm{L}$ \\
\hline
\end{tabular}

Fuente: Elaboración propia *Ver Tabla 1

\section{Sobre las Competencias de los Directivos de} las III.EE.

Para determinar cuáles deberían ser las competencias de los directivos, comparamos lo temas planteados por el MINEDU en sus documentos en los que hacen referencia a la gestión (Ver Tabla 2) con los Programas de Formación y Capacitación de Directores y Subdirectores de II.EE. Públicas del MINEDU que se muestra a continuación: 
Tabla 3

\begin{tabular}{|c|c|c|c|c|}
\hline \multirow{2}{*}{$\frac{\text { Módulo }}{T}$} & \multicolumn{2}{|c|}{ Nontre del Módulos } & \multirow{2}{*}{$\frac{\begin{array}{c}\text { Driración } \\
\text { (días) }\end{array}}{5}$} & \multirow{2}{*}{$\begin{array}{c}\begin{array}{c}\text { Hotas } \\
\text { Cronológicas }\end{array} \\
40\end{array}$} \\
\hline & $\begin{array}{l}\text { Desarrotlo } \\
\text { Profesional }\end{array}$ & Personal & & \\
\hline I] & \multicolumn{2}{|c|}{$\begin{array}{l}\text { Jiderargo Pedagógico y Políticas } \\
\text { de Gestión Iiscolar }\end{array}$} & 3 & 24 \\
\hline IJJ. & \multicolumn{2}{|c|}{$\begin{array}{l}\text { Cotopromisos e indicadores de } \\
\text { Gestión escolar }\end{array}$} & 3 & 24 \\
\hline ГV & \multicolumn{2}{|c|}{ Plan Anual de Trabajo } & 4 & 32 \\
\hline & \multicolumn{2}{|c|}{ Trotal } & 15 & 120 \\
\hline
\end{tabular}

Fuente: MINESOU

Contrastando estos módulos con las competencias que se requieten y que muestran los documentos analizados del MINEDU, es posible que la capacitación no esté orientada adecuadamente para la formación de directivos en educación, debido a que existen temas más específicos a desartollar. La situación es más preocupante aun si en la formación en educación en el nivel de pregrado, no se inciden en competencias directivas. Sería necesario establecer, en el perfil para los cargos directivos, la acreditación de competencias en temas de gestión - administración en el nivel de postgrado, con el fin de logtar resultados adecuados, de lo contratio las expresiones referidas en sus documentos sobre gestión quedarán sólo en literatura y, por ende, seguiremos hablando de gestión sin aterrizar en estrategias concretas para la formación de directivos en educación.

Asimismo, también debemos reflexionar sobre la forma y el contenido de las evaluaciones a directores y subdirectores que realiza el MINEDU. Estas no podrian resumirse a la validación de currículum vitae o a un examen para la comprensión de textos funcionales o a una prueba de solución de casos (RM 270-2014-MNNEDU), sino a la identificación de habilidades directivas cuyas características propias no son visibles en el curtículo ni en la evaluación. Por ello, se propone incidir para la evaluación de estos cargos (al finalizar su periodo) en los resultados o en el concepto de Ptesupuesto por Resultados (PpR), establecido por el Ministerio de Economía y Finanzas (MEF).

Este perfil y evaluación por tesultados podría constituit la siguiente fase estratégica del MINEDU en su plan de otorgamiento de cargos directivos por meritocracia.

Asimismo, el Presupuesto por Resultados (PpR) tiene relación con la calidad del gasto público, más aún si actualmente la política educativa en el Perú, es el de incrementar el gasto en educación, justificablemente; sin embargo, no podriamos garantizar la calidad del gasto si los directivos no tienen las competencias para haceto, y si no se establecen mecanismos de control adecuados para medir; en primer lugar, los resultados de la gestión pedagógica (liderazgo pedagógico); en segundo lugar, los logtos y mejoras concretas en los procesos vinculados con la enseñanza y el aprendizaje; y en tercerlugarlos resultados delagestión administrativa. Este escenario no podría darse si el MINEDU no establece estrategias de Empowerment (delegar poder) para tomar decisiones en las II.EE. que permitan el cambio en la educación, y si no se simplifica la normativa relacionada con lagestión educativa.

\section{La Gestión educativa versus las competencias transversales de los Directivos en Educación}

La gestión educativa no se limita a la ejecución u operatividad de los planes y programas educativos, sino se asegura que esta ejecución debe estar acompañada con el desarrollo de competencias de Ios directivos en temas transversales que juegan un rol importante como eje fundamental en el desartollo de la comunidad educativa. Estas competencias están vinculadas con los siguientes temas:

- Sostenibilidad, cambio dimático, biodiversidad, riesgo y desastres

La UNESCO (2014) pone de manifiesto las nuevas perspectivas del desarrollo humano sostenible en la última Conferencia Mundial sobre educación.

\section{- Creatividad e innovación}

I.eon Tratehtemberg (2014), importante especialista en temas educativos en el Perú, manifiesta "(..) Es dificil cambiar y actualizar la profesión de la educación" "... no se entiende que las cosas se pueden hacer de otra manera." 


\section{UNIFÉ - EPG}

- Teoría de la Complejidad Morín (1994 pág. 32) afirma que “(...) es un tejido de constituyentes heterogéneos inseparables asociados: presenta la paradoja de lo uno y lo múltiple (...)".

Al respecto, las organizaciones educativas son complejas, debido a que la comunidad educativa está integrada por directivos, docentes, alumnos y padres de familia, cada uno en una concepción de pensamiento complejo, por lo que, se requiere que el directivo tenga una visión mucho más cercana y real de las II.EE., situación que ayudaría a personalizar la educación, la inclusión y la formación integral. Es una comprensión del mundo en el que todo se encuentra conectado.

- Teoría de los Sistemas

Las organizaciones educativas como las demás organizaciones tienen un sistema abierto, ya que están en contacto con su entorno y cualquier variación en un subsistema podría afectar el sistema. También debemos considerar que el sistema de una II.EE. es parte de un macro sistema (global); por tanto, los directivos no pueden ser ajenos a los cambios disruptivos que se producen permanentemente en los macro sistemas.

\section{- Estrategia}

Minzberg (1998, p. 51), menciona que "[...] integra las principales metas y políticas de una organización $y$, a la vez, establece la secuencia coherente de las acciones a realizar $[\ldots .$. "

Por tanto, la revalorización de la carrera docente $y$, por ende, de los directivos en educación, concierne sólo a estos, en tanto, demuestren las competencias docentes y directivas, acompañadas de motivaciones intrínsecas para su desempeño y luego la revalorización económica del Estado como una carrera tan importante como las ingenierías, la administración de empresas, la medicina o el derecho.

\section{Conclusiones}

1. La gestión concebida desde la perspectiva del sistema educativo del Perú, presentada en diversos documentos relevantes, que son de conocimiento y uso de los directivos en educación, contienen una serie de temas que exigen competencias específicas que tendrían que desarrollar estos directivos; sin embargo, por la hegemonía de la especialidad en educación, no se podría garantizar que estas se puedan cumplir como parte de las responsabilidades asignadas.

2. La gestión es propia de la especialidad de la ciencia de la administración, por tanto, es necesario guardar coherencia en dos aspectos (1) entre los contenidos explícitos en los documentos relacionados del MINEDU y las competencias reales de los actuales directivos para asumir este tipo de responsabilidad y (2) entre los contenidos de los temas en la capacitación de directivos y los que contienen los documentos del MTNEDU para la gestión de II.EE.

3. La gestión como la administración se sustenta en los principios de esta; por ello, la gestión no podría desarrollarse si no existe un aprendizaje de su proceso fundamental: Planeación, Organización, Dirección y Control, así como otras concepciones actuales, cuyas características complejas, son. secuenciales, cíclicas, dinámicas e interactivas para cualquier organización.

4. Los documentos con contenidos de gestión del MINEDU evidencian temas (Ver Cuadro $\mathrm{N}^{\circ}$ 2) en los que se debe incidir en la capacitación de directivos, ya que la adquisición de estas competencias serían más acordes con los objetivos y resultados que se esperan en educación. Todas estas competencias deben ser orientadas a la gestión pedagógica.

5. La evaluación de directivos debe reorientarse a observar resultados en nuevos escenarios de creatividad y de innovación educativa cambios positivos de la realidad en las II.EE. que garanticen la calidad del gasto, situación que puede presentarse si se asegura un nuevo perfil del directivo que evidencie formación gerencial o directiva, observable, más allá de la validación del currículum vitae o a un examen para la comprensión de textos funcionales o una prueba de solución de casos. 
6. La gestión educativa no puede ser dispensada de competencias transversales, que deben tener los directivos. Algunas de estas, están propuestas en organizamos internacionales en su preocupación por la calidad de la educación como Sostenibilidad y Cambio Climático, Riesgos y Desastres, Creatividad e Innovación, Teoria de la Complejidad, Teoría de Sistemas y Estrategia para la Educación.

\section{Referencias}

Chiavenato, 1. (2004). Introductión a la Teoria General de la Administración. ( $7^{\circ}$ ed.) México D.F. México: McGtaw- Hill.

Definición. (2015). Concepto de Gestion, Recuperado de http://definicion.de/gestion/

Koontz, H. y Weihrich, H. (1997). Administracion-Una Perspettiva Global (10 ed.) México D.F. México: McGraw- Hill.

León Tratehtemberg. (2014) La Educación en el Perí ${ }_{2}$ Hacia dónde vamos? Recuperado de https:// wwwyoutube $\mathrm{com} /$ watch? $\mathrm{v}=\mathrm{DAzbPrV4} \mathrm{po}$

MINEDU. (2001). Marco operatorio. Gestión Pedagógica, En Diseño Curricular Básico de Educación (Adolescentes) Lima: DINESST - UDECREES. Recuperado de http://www.minedu. gob.pe/gestion_pedagogica/dir_edu_ secun_tecnologica/cambio_curricular/ curti2001/completo.htm

MINEDU. (2001). Manual de Redes Educativas Rurales - Un modelo de gestión para la educación rural en el Perú, Lima - OAAEUDECE, MECEP-BIRF

MINEDU. (2003). Cuaderno de Trabajo para la gestión Descentralizada de la Educación (2013), Especialidad Comunicación Secundaria. Recuperado de http://wwwminedu.gob.pe/p/ xtras/la-gest $\%$ CC3\%B3n-descentralizada-de-laeducacion.pdf

MINEDU. (2003). Cutrículo de Formación Docente Especialidad Comunicación Secundaria, Recuperado de http://www.
Alétheia 2015,3(1) 27 minedu.gob.pe/normatividad/reglamentos/ xtras/curt_com_sec.pdf

MINEDU. (2014). Fascículo: Gestión Escolar Centrada en los Aprendizajes. Recuperado de http://www.minedu.gob.pe/n/xtras/ fasciculo_gestion_escolar_centrada_en_ aprendizajes.pdf

MINEDU. (2014). Resolución Ministerial No 270-2014-MINEDU - Norma Técnica denominada "Normas para la evaluación excepcional prevista en la Décima Primera Disposición Complementaria Transitoria del Reglamento de la Ley de Reforma Magisterial" Organización del Procedimiento de Evaluación Perú. pág. 2.

Minztberg, H. y Brian, J. (1998). Proceso Estratégico, Toronto, Canada: Prentice Hall Hispanoamérica S.A.

Real Academia Española (REA) (2014). Diccionario de la Lengua Española. (23va ed.) Recuperado de http://lema.rae.es/drae/?val=gestion

Robbins, Stephen y De Cenzo, David (2003) Fundamentos de Administración, Conceptos y Aplicaciones. México. Prentice Hall.

Significados (2015) Significado de Gestión. Recuperado de http://www.significados.com/gestion/

UNESCO, (2014) Conferencia Mundial sobre educación, Japón - UNESCO World Conference on Education for Sustainable Development Learning Today for a Sustainable Future. 